\title{
Treatment patterns of knee osteoarthritis patients in Korea
}

\author{
Ha-Rim Park ${ }^{1, \star}$, Soo-Kyung Cho ${ }^{1, \star}$, Seul Gi Im², Sun-Young Jung ${ }^{3}$, Dalho Kim² ${ }^{2}$, Eun Jin Jang ${ }^{4}$, and \\ Yoon-Kyoung Sung ${ }^{1}$
}

\begin{abstract}
${ }^{1}$ Department of Rheumatology, Hanyang University Hospital for Rheumatic Diseases, Seoul; ${ }^{2}$ Department of Statistics, Kyungpook National University, Daegu; ${ }^{3}$ Chung-Ang University Collage of Pharmacy, Seoul; ${ }^{4}$ Department of Information Statistics, Andong National University, Andong, Korea
\end{abstract}

Received: August 31, 2017

Revised : October 12, 2017

Accepted: October 16, 2017

\section{Correspondence to}

Yoon-Kyoung Sung, M.D.

Department of Rheumatology,

Hanyang University Hospital for

Rheumatic Diseases, 222-1

Wangsimni-ro, Seongdong-gu,

Seoul 04763 , Korea

Tel: $+82-2-2290-9250$

Fax: +82-2-2298-8231

E-mail: sungyk@hanyang.ac.kr

*These authors contributed equally to this work.
Background/Aims: To evaluate the treatment patterns of knee osteoarthritis (OA) patients in South Korea.

Methods: Using the Korean nationwide claims database, all knee OA patients in Korea during 2014 were identified by the knee OA diagnostic code (M17) or any OA diagnostic code (M15 to M19) in combination with a procedure for a knee X-ray. Patterns of medications such as nonsteroidal anti-inflammatory drugs (NSAIDs), corticosteroids (CSs), analgesics, and symptomatic slow acting drugs for OA (SYSADOA) were analyzed. Prevalence and characteristics of knee OA patients who received a CS intra-articular injection (IAI) were also evaluated.

Results: We identified 2,016,516 knee OA patients whose age (mean \pm standard deviation) was $63.2 \pm 10.8$ years. The number of patients with at least one use of NSAIDs, analgesics, CS, and SYSADOA were $82.5 \%, 32.2 \%$, 8.6\%, and $43.4 \%$, respectively. The use of herbal SYSADOAs was $29.7 \%$. For regular users (medication possession ratios $\geq 50 \%$ ), the use of NSAIDs was substantially decreased (48.8\%), while the use of SYSADOA (37.3\%) and CS (6.7\%) were not significantly changed. The number of CS IAI users among knee OA patients was $0.18 \%$; they were slightly older $(64.4 \pm 10.9$ vs. $63.2 \pm 10.8, p<0.01)$ and more skewed towards females $(75.7 \%$ vs. $71.5 \%, p<0.01)$ than patients who had not received CS IAI.

Conclusions: In Korea, the use of SYSADOA or CS in knee OA patients was relatively high. Further studies on the effectiveness and the safety of these treatment options for knee OA are needed.

Keywords: Knee; Osteoarthritis; Therapeutics

\section{INTRODUCTION}

Osteoarthritis (OA), the most common form of arthritis, is a leading cause of disability and has a great impact on public health [1]. With the aging of the population and rising rates of obesity, the prevalence of knee $\mathrm{OA}$ is increasing [2]. Roughly $25 \%$ of adults aged 55 years or older have knee pain on most days, and most of these people have OA [3]. In Korea, the prevalence of radiologic OA and symptomatic OA was reported as $37.3 \%$ and $24.2 \%$, respectively, in an elderly community residents' survey [4]. In a recent report using the Fifth Korean National Health and Nutrition Examination Survey (2010 to 2012), the number of knee OA patients with symptoms and radiological findings in males and females was $4.5 \%$ and $19 \%$, respectively [5].

To date, there is no known cure for OA, and management of knee OA patients remains palliative treatment. Several therapies are available to reduce OA-associated symptoms. The most commonly used treatment op- 
tions are focused on pain relief and improvement of joint function, which include nonsteroidal anti-inflammatory drugs (NSAIDs), analgesics (acetaminophen or opioids), and symptomatic slow acting drugs for osteoarthritis (SYSADOA). These drugs are recommended in many knee OA guidelines [6-10]. In addition, corticosteroid (CS) intra-articular injection (IAI) can be a treatment option for knee OA. However, consensus on therapeutic strategies is inadequate and guidance on first-line therapies or drug combination is poorly implemented. This leads to great variability in the use of different drugs for knee OA in clinical practice. In clinical practice, physicians usually choose a treatment for knee OA by considering the treatment's effects and adverse events depending on the patient's condition irrespective of health care costs and patient safety [11].

This study aimed to evaluate the treatment patterns of knee OA patients in South Korea.

\section{METHODS}

\section{Data source and study population}

Korean Health Insurance Review and Assessment Service database

South Korea has a universal health coverage system in which the National Health Insurance program covers almost $100 \%$ of the Korean population, approximately 50 million people. Korean Health Insurance Review and Assessment Service (HIRA) database contains individual beneficiary information, in addition to healthcare service information such as diagnosis, procedures, prescriptions, and the type of institution or department [12].

\section{Study population}

On the basis of our previous validation study [13], knee OA patients were identified by using the knee OA diagnostic code $\left(\mathrm{M}_{17}\right)$ or any OA diagnostic code $\left(\mathrm{M}_{15}\right.$ to M19) in combination with a procedure for a knee X-ray in the same claim. After excluding patients with inflammatory arthritis such as rheumatoid arthritis (RA), ankylosing spondylitis (AS), or psoriatic arthritis, we constructed a database of patients $\geq 40$ years of age with knee OA (Fig. 1). To evaluate healthcare usage rates of knee OA patients, the type of institution and depart- ment frequently visited during the follow-up period was analyzed. For all knee OA patients in 2014, the number of comorbidities and the Charlson comorbidity index (CCI) was tabulated.

\section{Treatment patterns of knee OA}

Oral medications for knee OA patients

Medications used in our study included oral NSAIDs, analgesics such as acetaminophen, tramadol, and their combination. Well-known SYSADOAs such as diacerein, avocado soybean unsaponifiables (ASU), and chondroitin were included in this study, but glucosamine was excluded since it was reimbursed by HIRA only since 2011. Instead, herbal drugs combining herbal extracts such as herbal SYSADOA A [14] (a mixed extract of three herbs, Clematis mandshurica, Prunella vulgaris, and Trichosanthes kirilowii), herbal SYSADOA B $[15,16]$ (an ethanol extract prepared from 12 plant sources, Chaenomelis fructus, Achyranthis radix, Acanthopanax cortex, Cinnamomi cortex, Gentianae macrophyllae radix, Clematidis radix, Angelica gigantis radix, Cnidii rhizoma, Gastrodiae rhizoma, Carthami flos, Saposhnikoviae radix, and Dipsaci radix), and herbal SYSADOA C [17] (a purified extract from a mixture of six oriental herbs, Ledebouriellae radix, Achyranthis radix, Acanthopanacis cortex, Cibotii rhizoma, Glycine semen, and Eucommiae cortex). In addition, the use of oral CS was also investigated.

\section{Utilization of oral medications}

Drug utilization was described in terms of prevalence of use, which was defined by the number of doses at any time during follow-up. Quantitative use of each different medication was described using medication possession ratios (MPRs). The MPR is a standard measurement that is defined as the number of days for which medication is available divided by the number of days of treatment (time from first to last prescription) [15]. Regular use of a specific medication was defined as a MPR of $50 \%$, and occasional use of a specific medication was defined as a MPR of $25 \%$. The number and the types of drugs used during the follow-up period were estimated.

Utilization rates of corticosteroid intra-articular injection For evaluating the utilization rates of CS IAI, we counted claims with a knee OA diagnostic code that also includ- 
ed the procedure code of CS IAI within the same claim. The frequency of CS IAI was also evaluated according to age and gender.

\section{Statistical analysis}

Baseline characteristics of the study population were presented by demographics, medical care utilization (including type of institution or department), and comorbidities that were most frequently observed during the follow-up period. We used descriptive analysis to examine the drug utilization in knee OA patients. Drugs were categorized into four categories: NSAIDs, analgesics, SYSADOAs, and CS. The usage frequency of each drug and combinations of each category of drugs were calculated. $p$ values less than 0.05 were considered statistically significant. All of the analyses were performed using SAS software version 9.3 (SAS Institute Inc., Cary, NC, USA).

\section{Ethics statement}

The Institutional Review Board (IRB) of Hanyang University determined that this study was exempt from IRB review because we used existing data and the information could not be linked to individual subjects (HYUH2016-06-008-002). Informed consent was not required because data was de-identified and collected retrospectively.

\section{RESULTS}

\section{Baseline characteristics}

We identified 2,016,516 knee OA patients from 2014. Their mean age \pm standard deviation was $63.2 \pm 10.8$ years and $71.5 \%$ were female (Table 1). Most of the patients went to a private clinic $(\mathrm{n}=1,310,406,65 \%)$ or a community hospital ( $\mathrm{n}=354.391,17.6 \%)$. More than half of the patients (72.6\%) visited an orthopedic surgery department. Nearly half of the patients had at least two comorbidities ( $\mathrm{n}=972,546,48.2 \%)$. The most frequent comorbidity was chronic pulmonary disease $(n=692,582$, $34.3 \%)$, followed by peptic ulcer disease $(n=656,114$, $32.5 \%)$ and mild liver disease $(n=492,225,24.4 \%)$. The mean CCI score was $2.56 \pm 1.78$.
Table 1. Baseline characteristics of patients with knee osteoarthritis

\begin{tabular}{|c|c|}
\hline Characteristic & Value \\
\hline Age & $63.2 \pm 10.8$ \\
\hline Female sex & $1,442,510(71.5)$ \\
\hline \multicolumn{2}{|l|}{ Type of institution ${ }^{\mathrm{a}}$} \\
\hline Tertiary hospital & $53,574(2.7)$ \\
\hline General hospital & $171,826(8.5)$ \\
\hline Community hospital & $354,391(17.6)$ \\
\hline Clinic & $1,310,406(65.0)$ \\
\hline Others & $126,319(6.3)$ \\
\hline \multicolumn{2}{|l|}{ Type of department ${ }^{\mathrm{a}}$} \\
\hline Internal Medicine & $138,183(6.9)$ \\
\hline Orthopedic Surgery & $1,464,264(72.6)$ \\
\hline Rehabilitation & $38,295(1.9)$ \\
\hline Others & $375,774(18.6)$ \\
\hline \multicolumn{2}{|l|}{ No. of comorbidities } \\
\hline 1 & $546,605(27.1)$ \\
\hline$\geq 2$ & $972,546(48.2)$ \\
\hline Myocardial infarction & $21,943(1.1)$ \\
\hline Congestive heart failure & $113,620(5.6)$ \\
\hline Peripheral vascular disease & $364,022(18.1)$ \\
\hline Cerebrovascular disease & $243,677(12.1)$ \\
\hline Dementia & $117,794(5.8)$ \\
\hline Chronic pulmonary disease & $692,582(34 \cdot 3)$ \\
\hline Peptic ulcer disease & $656,114(32.5)$ \\
\hline Mild liver disease & $492,255(24 \cdot 4)$ \\
\hline Diabetes without chronic complication & $485,567(24.1)$ \\
\hline Diabetes with chronic complication & $172,641(8.6)$ \\
\hline Hemiplegia or paraplegia & $20,932(1.0)$ \\
\hline Renal disease & $34,275(1.7)$ \\
\hline Any malignancy & $112,417(5 \cdot 6)$ \\
\hline Moderate or severe liver disease & $8,244(0.4)$ \\
\hline Metastatic solid tumor & $9,175(0.5)$ \\
\hline Charlson comorbidity index score & $2.6 \pm 1.8$ \\
\hline
\end{tabular}

Values are presented as mean \pm SD or number (\%).

${ }^{a}$ Type of institution and type of department frequently visited during the follow-up period. 
Table 2. Medication utilization in patients with knee osteoarthritis

\begin{tabular}{|c|c|c|c|}
\hline Characteristic & Any use $^{\mathrm{a}}$ & Occasional use $\mathrm{b}^{\mathrm{b}}$ & Regular use $^{\mathrm{c}}$ \\
\hline NSAIDs & $1,664,518(82.5)$ & $1,221,533(60.6)$ & $983,783(48.8)$ \\
\hline Nonselective NSAIDs & $1,429,388(70.9)$ & $1,127,672(55 \cdot 9)$ & $896,697(44.5)$ \\
\hline Cox-2 inhibitor & $80,853(4.0)$ & $77,107(3.8)$ & $70,578(3.5)$ \\
\hline SYSADOA & $875,854(43.4)$ & $821,013(40.7)$ & $751,391(37 \cdot 3)$ \\
\hline Diacerein & $334,761(16.6)$ & $297,476(14.8)$ & $258,255(12.8)$ \\
\hline Avocado soybean unsaponifiables & $165,010(8.2)$ & $157,014(7.8)$ & $143,913(7.1)$ \\
\hline Chondroitin & $45,307(2.3)$ & $41,665(2.1)$ & $37,426(1.9)$ \\
\hline Herbal SYSADOA B & $158,577(7 \cdot 9)$ & $147,145(7 \cdot 3)$ & $132,654(6.6)$ \\
\hline Herbal SYSADOA A & $324,677(16.1)$ & $299,697(14 \cdot 9)$ & $268,620(13 \cdot 3)$ \\
\hline Herbal SYSADOA C & $114,258(5 \cdot 7)$ & $108,519(5.4)$ & $99,801(5.0)$ \\
\hline Analgesics & $648,611(32.2)$ & $559,316(27.7)$ & $478,199(23.7)$ \\
\hline Acetaminophen & $75,423(3.7)$ & $63,553(3.2)$ & $57,307(2.8)$ \\
\hline Tramadol & $20,212(1.0)$ & $19,162(1.0)$ & $17,622(0.9)$ \\
\hline Acetaminophen + Tramadol & $593,581(29 \cdot 4)$ & $505,292(25.1)$ & $424,539(21.1)$ \\
\hline Corticosteroid & $173,591(8.6)$ & $150,419(7.5)$ & $134,294(6.7)$ \\
\hline
\end{tabular}

Values are presented as number (\%).

NSAID, nonsteroidal anti-inflammatory drug; Cox-2, cyclooxygenase 2; SYSADOA, symptomatic slow-acting drug in osteoarthritis.

${ }^{a}$ Any use was defined as any number of doses at any time during follow-up.

${ }^{\mathrm{b}}$ Occasional use was defined as a medication possession ratio (MPR) of $25 \%$.

${ }^{\mathrm{c}}$ Regular use was defined as a MPR of $50 \%$.

\section{Patterns of oral medications in knee OA patients}

The prevalence of oral medications for knee OA patients Among the total patients, $12.0 \%$ of patients did not use any medications for 1 year. The medication used most frequently at any time during follow-up was NSAIDs (82.5\%), followed by SYSADOA (43.4\%) and analgesics (32.2\%). Among SYSADOAs, diacerein (16.6\%) and herbal SYSADOA A (16.1\%) were the most commonly used, followed by ASU (8.2\%) and herbal SYSADOA B (7.9\%). The percentage of total herbal SYSADOAs were high as 29.7\%. Among patients who used analgesics, tramadol/ acetaminophen combination product was the most frequently used (29.4\%), while single use of acetaminophen was only $3.7 \%$. Oral CS was used in $8.6 \%$ of patients (Table 2).

Quantitative drug utilization was estimated by taking the percentages of patients defined as occasional use $(\mathrm{MPR} \geq 25 \%)$ and regular use (MPR $\geq 50 \%)$ for each drug. The data is presented in Table 2. In this quantitative drug utilization, percentage of drug use was decreased along the MPRs for occasional use and regular use. When we analyzed a group of regular users, the use of NSAIDs was substantially decreased from $82.5 \%$ to $48.8 \%$, while the use of SYSADOAs (37.3\%) and analgesics (23.7\%) was not significantly different. The regular use (MPR $\geq 50 \%$ ) of oral CS was also relatively high at $6.7 \%$ (Table 2).

\section{The number of drugs patients experienced during follow-up}

More than half of knee OA patients were treated with drugs from at least two categories within a calendar year: $37.2 \%$ of patients were treated with drugs from two categories and $16.6 \%$ were treated with drugs from three categories. NSAIDs alone were the most common treatment pattern (27.2\%), although analgesics alone or SYSADOAs alone were also observed. Among patients treated with multiple drugs, NSAIDs in combination with SYSADOAs (22.3\%), or a combination of NSAIDs, SYSADOAs, and analgesics (13.0\%) were commonly observed. We also observed patients that were treated with drug combinations that did not include SYSADOAs: NSAIDs in combination with analgesics (11.9\%), NSAIDs in combination with CS (1.9\%), and a combination of NSAIDs, analgesics and CS (1.7\%) (Table 3). 


\section{Intra-articular injection of CS in knee OA patients}

A total of 3,567 patients (0.18\%) received CS IAI at least once in 2014. Patients who received CS IAI were slightly older than patients who did not receive IAI (64.4 \pm 10.9 vs. $63.2 \pm 10.8, p<0.01)$. Females more frequently

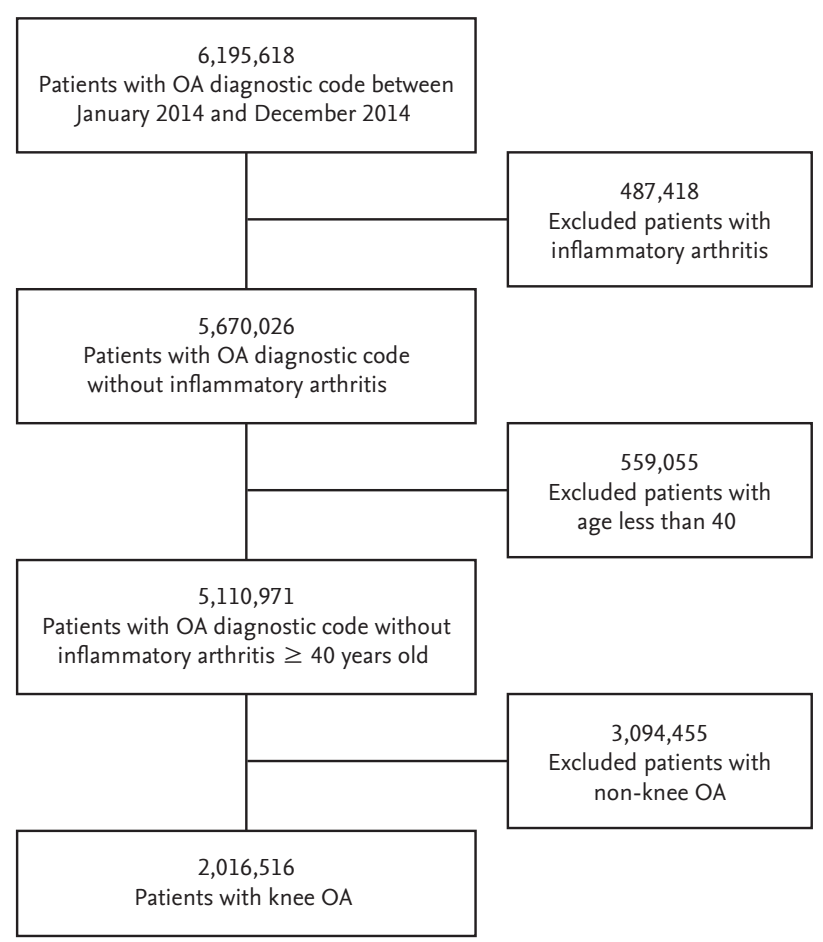

Figure 1. Patient selection flow. OA, osteoarthritis. received CS IAI (75.7\% vs. $71.5 \%, p<0.01$ ) (Supplementary Fig. $1 \mathrm{~A})$. However, the frequency of females was higher than males in patients that received only one or two times of IAI with CS while the frequency of males was higher in groups receiving over three times the number

Table 3. Number of drugs patients experienced by knee osteoarthritis patients during follow-up

\begin{tabular}{lc}
\hline Type of drug & No. $(\%)$ \\
\hline Single drug & $632,856(31.4)$ \\
\hline NSAIDs & $549,292(27.2)$ \\
SYSADOA & $47,190(2.3)$ \\
Analgesics & $33,111(1.6)$ \\
\hline Corticosteroid & $3,263(0.2)$ \\
Two drugs & $749,655(37.2)$ \\
\hline NSAIDs + SYSADOA & $448,932(22.3)$ \\
\hline NSAIDs + Analgesics & $239,089(11.9)$ \\
\hline NSAIDs + Corticosteroid & $37,275(1.9)$ \\
SYSADOA + Analgesics & $20,713(1.0)$ \\
\hline SYSADOA + Corticosteroid & $1,276(0.1)$ \\
\hline Analgesics + Corticosteroid & $2,370(0.1)$ \\
\hline Three drugs & $334,420(16.6)$ \\
\hline NSAIDs + SYSADOA + Analgesics & $261,800(13.0)$ \\
\hline NSAIDs + SYSADOA + Corticosteroid & $37,879(1.9)$ \\
\hline SYSADOA + Analgesics + Corticosteroid & $1,277(0.1)$ \\
\hline NSAIDs + Analgesics + Corticosteroid & $33,464(1.7)$ \\
\hline Other combinations & $56,787(2.8)$ \\
\hline SAID & \\
\hline
\end{tabular}

NSAID, nonsteroidal anti-inflammatory drug; SYSADOA, symptomatic slow-acting drug in osteoarthritis.

Table 4. Factors associated with risk of intra-articular steroid injection in patients with knee osteoarthritis ${ }^{\mathrm{a}}$

\begin{tabular}{|c|c|c|c|c|}
\hline \multirow{2}{*}{ Variable } & \multicolumn{2}{|c|}{ Crude } & \multicolumn{2}{|c|}{ Adjusted } \\
\hline & OR (95\% CI) & $p$ value & OR (95\% CI) & $p$ value \\
\hline Age, yr & $1.01(1.01-1.01)$ & $<0.01$ & $1.00(1.00-1.01)$ & 0.04 \\
\hline \multicolumn{5}{|l|}{$40-49$ (ref) } \\
\hline $50-59$ & $1.36(1.19-1.55)$ & 0.05 & & \\
\hline $60-69$ & $1.28(1.12-1.46)$ & 0.99 & & \\
\hline$\geq 70$ & $1.55(1.36-1.76)$ & $<0.01$ & & \\
\hline Female sex & $1.24(1.15-1.34)$ & $<0.01$ & $1.32(1.22-1.42)$ & $<0.01$ \\
\hline \multicolumn{5}{|c|}{ No. of comorbidity } \\
\hline 1 & $1.56(1.39-1.75)$ & 0.84 & $1.46(1.31,1.64)$ & 0.96 \\
\hline$\geq 2$ & $2.47(2.24-2.73)$ & $<0.01$ & $2.13(1.92-2.36)$ & $<0.01$ \\
\hline
\end{tabular}

OR, odds ratio; CI, confidence interval.

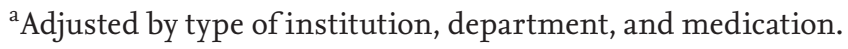


of IAIs with CS (Supplementary Fig. 1B). Comorbidities (CCI score, $3.1 \pm 2.1$ vs. $2.6 \pm 1.8, p<0.01$ ) and the use of multiple drug combinations (three types of drugs, $23.4 \%$ vs. $14.9 \%, p<0.01$ ) were greater in patients with CS IAI. In a multivariable logistic model adjusted by type of institution, type of department, female (odds ratio [OR], 1.3; $95 \%$ confidence interval [CI], 1.2 to 1.4 ) and the number of comorbidities (OR, 1.5; 95\% CI, 1.3 to 1.6 in patients with one comorbidity; OR, 2.1; 95\% CI, 1.9 to 2.4 in patients with more than two comorbidities) were associated with IAI with CS in patients with knee OA (Table 4).

\section{DISCUSSION}

In this study, we extracted a large dataset of knee OA patients $(\mathrm{n}=2,016,516)$ using a nation-wide Korean claims database and identified patterns of medication use for knee OA patients. Most of the patients (82.5\%) were treated with oral NSAIDs and nearly half of the patients (48.8\%) used NSAIDs regularly. The use of SYSADOAs, particularly herbal SYSADOAs, was commonly observed and SYSDOAs were frequently used in combination with NSAIDS. Unexpectedly, the use of oral CS was high in knee OA patients. On the other hand, CS IAI was not common. Female patients, older age, and the number of comorbidities were associated with CS IAI in knee OA patients.

We identified some unique features of medication use specific to Korean patients with knee OA that differed from medication use in other countries. First, the prevalence of NSAID use (82.5\%) in Korean patients was much higher than the $26.0 \%$ to $58.1 \%$ recently reported from the USA using osteoarthritis initiative (OAI) data [18]. Regular use of NSAIDs (48.8\%) was also much higher than the $14.4 \%$ of patients reported in Spain [11]. This pattern may be related to our data source, which was a claims database consisting of prescriptions from physicians. For instance, knee OA patients with mild symptoms can use over-the-counter drug by themselves, whereas patients with severe symptoms are treated with NSAIDs by physicians. Many patients treated with NSAIDs in our study were also treated with other drugs such as SYSADOAs, analgesics, or CS and this pattern reflected their uncontrolled symptoms. In Korea, patients more commonly used analgesics, such as acetaminophen or paracetamol, rather than NSAIDs as over the counter drugs for pain control [19]. Hence physicians tend to prescribe NSAIDs frequently rather than acetaminophen.

Second, the prevalence of SYSADOA use (43.4\%) was comparable to other countries. $27.5 \%$ to $54.0 \%$ in OAI [18] and its regular use (37.3\%) was $46.6 \%$ in a study from Spain [11]. However, the individual drug selection was different from other countries. In the United States and European countries, glucosamine or chondroitin was frequently used, while new herbal medications were frequently prescribed in Korea. Until recently, glucosamine and chondroitin were widely used in Korea for the general population and not limited to knee OA patients [20], even though it was covered by medical reimbursement. However, these compounds were excluded from Korean reimbursement guidelines based on the the Glucosamine/chondroitin Arthritis Intervention Trial (GAIT) [21] and their use has since dramatically decreased. A recent clinical trial suggested that a combined chondroitin sulfate/glucosamine treatment for painful knee OA was effective [22]; however, another meta-analysis reported that there was no evidence to support the use of glucosamine for knee OA [23]. Because of the controversy surrounding the effectiveness of glucosamine for knee OA patients, reimbursement has become difficult.

Finally, the use of oral CS was high in Korean patients with knee OA. Many factors contribute to the inflammatory process in OA, which include the synthesis and release of inflammatory cytokines into the joint environment by multiple tissues. Thus, CS can be a potent anti-inflammatory agent in OA treatments, although the effectiveness and safety of CS treatments are unclear [24]. Treatment guidelines typically recommend CS IAI, but not oral CS, for knee OA patients [9].

The extremely high use of oral CS in Korean knee OA patients can be explained by several factors. For example, patients with comorbidities such as COPD and asthma could have been managed with CS. NSAIDs could have been ineffective in some patients, or the patients had a contraindication for NSAIDs, and thus may have had CS prescribed. Also, some patients who had undifferentiated arthritis or inflammatory arthritis patients not satisfied RA, AS, or psoriatic arthritis criteria could have received an OA diagnostic code. Nevertheless, since the prevalence of CS use in knee OA patients was extremely 
high in this study, further studies are needed to investigate the possibility of CS abuse in this population.

There is controversy surrounding the use of CS IAI. A recent review [8] suggested that CS IAI may be associated with a moderate improvement in pain and a small improvement in physical function, although the quality of the evidence was poor. The 2013 guidelines from the American Academy of Orthopedic Surgeons did not provide any recommendations supporting or discouraging the use of CS IAI because the evidence was inconclusive [6]. On the other hand, the 2012 guidelines from the American College of Rheumatology conditionally recommended CS IAI for knee OA [7], Furthermore, both the 2014 National Institute for Health and Care Excellence guidelines [8] and the 2014 Osteoarthritis Research Society International (OARSI) guidelines [9] stated that CS IAI was appropriate for knee OA. In clinical practice, CS IAI is widely considered the most acutely effective nonsurgical treatment for $\mathrm{OA}$ and typically provides substantial pain relief. However, the effect was shortterm which was corroborated in all meta-analyses [8]. Some patients with knee OA do not respond to CS IAI, perhaps because they do not have a substantial inflammatory component to their OA pain. In our study, the prevalence of CS IAI use was not high (0.18\%), but the patients who received CS IAI were older and had higher numbers of comorbidities. This observation indicate that CS IAI is possibly prescribed for patients who do not tolerate or adhere to oral medicines. Also, there may not be other treatment options for these patients, because they do not tolerate knee replacement operations.

This study has several strengths. First, the large population size reduced selection bias and may be generalizable due to data drawn from a large nation-wide claims database. Second, this is the first study to present the utilization of a new herbal SYSADOA, which has been commonly prescribed in Korea. Third, quantifying the use of each different type of medication using MPR gave us the prevalence of regular users in knee OA patients, although this was a cross-sectional study design.

There are some limitations to this study. First, we could not estimate the usage of glucosamine which was not reimbursed as medicine, but as a health supplement. However, we included all drugs that were available in the clinic, which provided exact drug utilization at the national level. Second, there is a possibility that we did not include knee OA patients with mild symptoms. However, the total number of patients $(n=2,016,516)$ in our study was about $10 \%$ of the Korean population 40 years or older. The number of knee OA patients reported here was similar to the prevalence of knee OA previously reported. Therefore, our study population represented the total sub-population of knee OA patients in Korea. Third, the prevalence of intra-articular hyaluronic acid (HA) treatment was not assessed because of the variability of its patterns of use. The provision of an operational definition for this treatment in the claims database, and long-term study will provide information on the exact prevalence and sparing effect of NSAIDs and CS.

From this study, we have identified areas for future study. First, the long term safety related to NSAIDs use by knee OA patients should be evaluated, since NSAIDSs are typically used in the elderly who are also treated with other medications to manage associated comorbidities. Second, a review of the effectiveness of the continuous use of SYSADOAs is needed. Specifically, more evidence is needed to evaluate the effectiveness and safety of the new herbal medications because of lack of good evidences even though their high prevalence. Also, the safety of IAI with not only CS but also HA and their effects on decrease in the use of NSAIDs or analgesics is also an important research issue.

In Korea, the usage of SYSADOA or CS in knee OA patients was relatively high. Further studies on the effectiveness and safety of these treatment options for knee OA are needed.

\section{KEY MESSAGE}

1. The number of patients with at least one use of nonsteroidal anti-inflammatory drugs (NSAIDs), analgesics, corticosteroid (CS), and symptomatic slow acting drugs for osteoarthritis (SYSADOA) were $82.5 \%, 32.2 \%, 8.6 \%$, and $43.4 \%$, respectively. For regular users, the use of NSAIDs was substantially decreased (48.8\%), while the use of SYSADOA (37.3\%) and CS (6.7\%) were not significantly changed.

2. In Korea, the use of SYSADOA or corticosteroids in knee osteoarthritis (OA) patients was relatively high and the new herbal SYSADOAs were 
frequently prescribed.

3. The number of CS intra-articular injection users among knee OA patients was $0.18 \%$; they were slightly older and more skewed towards females.

\section{Conflict of interest}

No potential conflict of interest relevant to this article was reported.

\section{Acknowledgments}

This research was supported by a grant of the Korea Health Technology R\&D Project through the Korea Health Industry Development Institute (KHIDI), funded by the Ministry of Health and Welfare, Republic of Korea (grant number: $\mathrm{HC}_{15} \mathrm{C}_{33} 88$ ).

\section{REFERENCES}

1. Murray CJ, Vos T, Lozano R, et al. Disability-adjusted life years (DALYs) for 291 diseases and injuries in 21 regions, 1990-2010: a systematic analysis for the Global Burden of Disease Study 2010. Lancet 2012;380:2197-2223.

2. Nguyen US, Zhang Y, Zhu Y, Niu J, Zhang B, Felson DT. Increasing prevalence of knee pain and symptomatic knee osteoarthritis: survey and cohort data. Ann Intern Med 2011;155:725-732.

3. Peat G, McCarney R, Croft P. Knee pain and osteoarthritis in older adults: a review of community burden and current use of primary health care. Ann Rheum Dis 2001;60:91-97.

4. Kim I, Kim HA, Seo YI, Song YW, Jeong JY, Kim DH. The prevalence of knee osteoarthritis in elderly community residents in Korea. J Korean Med Sci 2010;25:293-298.

5. Park JH, Hong JY, Han K, et al. Prevalence of symptomatic hip, knee, and spine osteoarthritis nationwide health survey analysis of an elderly Korean population. Medicine (Baltimore) 2017;96:e6372.

6. Jevsevar DS. Treatment of osteoarthritis of the knee: evidence-based guideline, and edition. J Am Acad Orthop Surg 2013;21:571-576.

7. Hochberg MC, Altman RD, April KT, et al. American College of Rheumatology 2012 recommendations for the use of nonpharmacologic and pharmacologic therapies in osteoarthritis of the hand, hip, and knee. Arthritis Care Res (Hoboken) 2012;64:465-474.

8. National Clinical Guideline Centre for Acute and Chronic Conditions (Great Britain); National Institute for Health and Care Excellence (Great Britain); National Collaborating Centre for Chronic Conditions (Great Britain). Osteoarthritis: Care and Management in Adults. London (UK): National Clinical Guideline Centre, 2014.

9. McAlindon TE, Bannuru RR, Sullivan MC, et al. OARSI guidelines for the non-surgical management of knee osteoarthritis. Osteoarthritis Cartilage 2014;22:363-388.

10. Jordan KM, Arden NK, Doherty M, et al. EULAR Recommendations 2003: an evidence based approach to the management of knee osteoarthritis: report of a Task Force of the Standing Committee for International Clinical Studies Including Therapeutic Trials (ESCISIT). Ann Rheum Dis 2003;62:1145-1155.

11. Wilson N, Sanchez-Riera L, Morros R, et al. Drug utilization in patients with OA: a population-based study. Rheumatology (Oxford) 2015;54:860-867.

12. Kim L, Kim JA, Kim S. A guide for the utilization of Health Insurance Review and Assessment Service National Patient Samples. Epidemiol Health 2014;36:e20140o8.

13. Park HR, Cho SK, Im SG, Jung SY, Jang EJ, Sung YK. Identification of knee osteoarthritis patients in the claims database. The 19th Asia Pacific League of Associations for Rheumatology Congress (APLAR 2017); 2017 Oct 16-20; Dubai, UAE.

14. Hartog A, Hougee S, Faber J, et al. The multicomponent phytopharmaceutical SKI306X inhibits in vitro cartilage degradation and the production of inflammatory mediators. Phytomedicine 2008;15:313-320.

15. Yoo WH, Yoo HG, Park SH, et al. Efficacy and safety of PG201 (Layla(R)) and celecoxib in the treatment of symptomatic knee osteoarthritis: a double-blinded, randomized, multi-center, active drug comparative, parallel-group, non-inferiority, phase III study. Rheumatol Int 2014;34:1369-1378.

16. Shin SS, Jin M, Jung HJ, et al. Suppressive effects of PG2O1, an ethanol extract from herbs, on collagen-induced arthritis in mice. Rheumatology (Oxford) 2003;42:665-672.

17. Lee SY, Kwon HK, Lee SM. SHINBARO, a new herbal medicine with multifunctional mechanism for joint disease: first therapeutic application for the treatment of osteoarthritis. Arch Pharm Res 2011;34:1773-1777.

18. Lapane KL, Liu SH, Dube CE, Driban JB, McAlindon TE, 
Eaton CB. Factors associated with the use of hyaluronic acid and corticosteroid injections among patients with radiographically confirmed knee osteoarthritis: a retrospective data analysis. Clin Ther 2017;39:347-358.

19. Chae MK, Bang JS, Lee YJ. Patterns of over-the-counter drug use and interactions between over-the-counter drugs and prescription drugs in adults visiting a community pharmacy. Korean J Clin Pharm 2013;23:49-56.

20. Seo HJ, Sung YK, Choi CB, et al. Prevalence and factors affecting glucosamine use in Korea: a survey-based study. Rheumatol Int 2013;33:1627-1631.

21. Clegg DO, Reda DJ, Harris CL, et al. Glucosamine, chondroitin sulfate, and the two in combination for painful knee osteoarthritis. N Engl J Med 2006;354:795-808.

22. Hochberg MC, Martel-Pelletier J, Monfort J, et al. Combined chondroitin sulfate and glucosamine for painful knee osteoarthritis: a multicentre, randomised, double-blind, non-inferiority trial versus celecoxib. Ann Rheum Dis 2016;75:37-44.

23. Runhaar J, Rozendaal RM, van Middelkoop M, et al. Subgroup analyses of the effectiveness of oral glucosamine for knee and hip osteoarthritis: a systematic review and individual patient data meta-analysis from the OA trial bank. Ann Rheum Dis 2017;76:1862-1869.

24. da Costa BR, Hari R, Juni P. Intra-articular corticosteroids for osteoarthritis of the knee. JAMA 2016;316:2671-2672. 


\section{KJIM'}
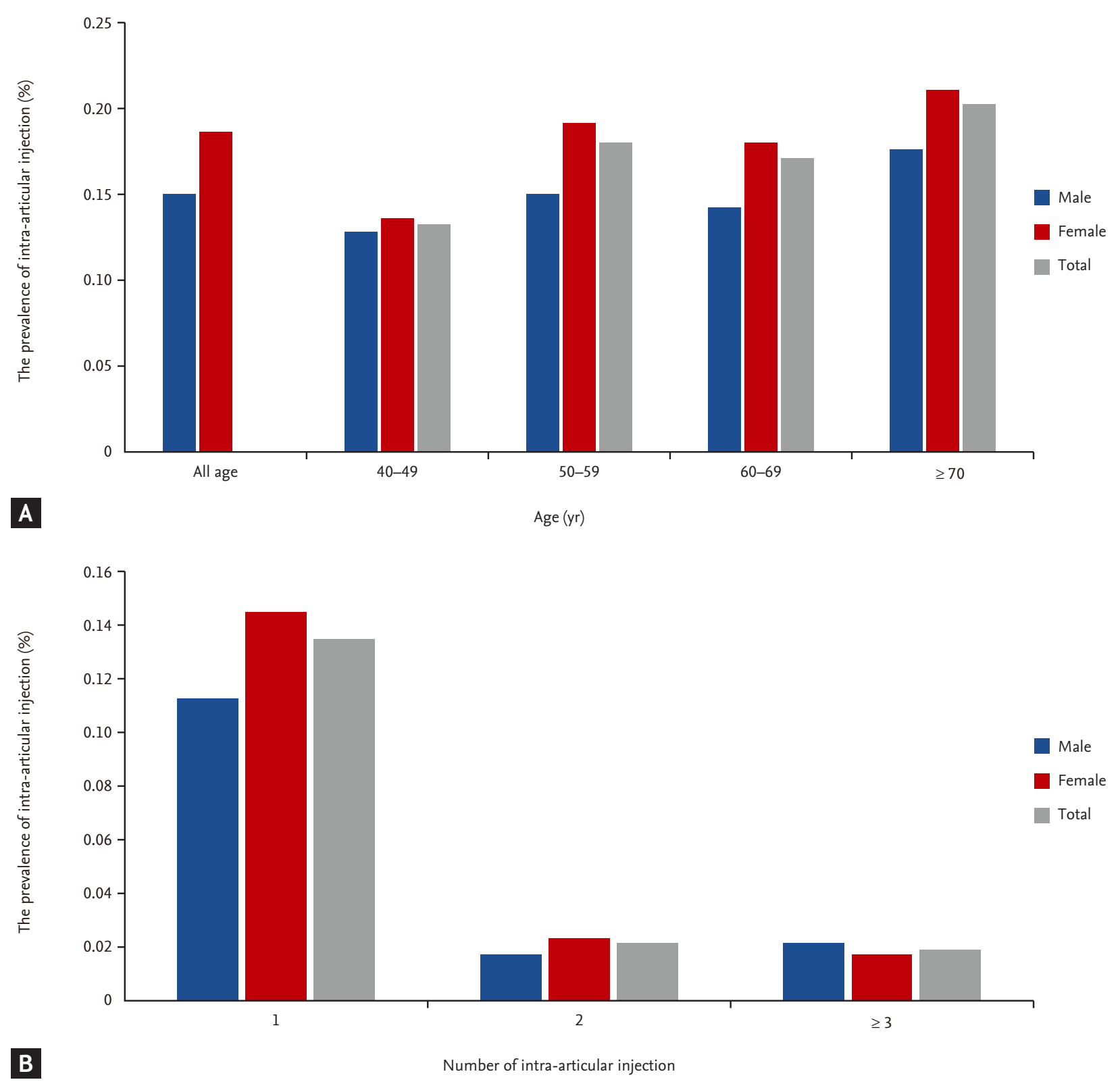

Supplementary Figure 1. Intra-articular injection with corticosteroids in knee osteoarthritis (OA) patients. (A) The usage of corticosteroid (CS) intra-articular injection (IAI) in knee OA patients. (B) The usage of CS IAI in knee OA patients per calendar year. 